\title{
Effect of thermospheric contraction on remediation of the near-Earth space debris environment
}

\author{
Hugh G. Lewis, ${ }^{1}$ Arrun Saunders, ${ }^{1}$ Graham Swinerd, ${ }^{1}$ and Rebecca J. Newland ${ }^{1}$ \\ Received 19 January 2011; revised 6 May 2011; accepted 16 May 2011; published 10 August 2011.
}

[1] Historically, computer simulations of the near-Earth space debris environment have provided a basis for international debris mitigation guidelines and, today, continue to influence international debate on debris environment remediation and active debris removal. Approximately 22,500 objects larger than $10 \mathrm{~cm}$ are known to exist in Earth orbit, and less than $5 \%$ of these are operational payloads, with the remaining population classed as space debris. These objects represent a significant risk to satellite operations because of the possibility of damaging or catastrophic collisions, as demonstrated by the collision between Iridium 33 and Cosmos 2251 in February 2009. Indeed, recent computer simulations have suggested that the current population in low Earth orbit (LEO) has reached a sufficient density at some altitudes for collision activity there to continue even in the absence of new launches. Even with the widespread adoption of debris mitigation guidelines, the growth of the LEO population, in particular, is expected to result in eight or nine collisions among cataloged objects in the next 40 years. With a new study using the University of Southampton's space debris model, entitled DAMAGE, we show that the effectiveness of debris mitigation and removal strategies to constrain the growth of the LEO debris population could be more than halved because of a long-term future decline in global thermospheric density. However, increasing debris remediation efforts can reverse the impact of this negative density trend.

Citation: Lewis, H. G., A. Saunders, G. Swinerd, and R. J. Newland (2011), Effect of thermospheric contraction on remediation of the near-Earth space debris environment, J. Geophys. Res., 116, A00H08, doi:10.1029/2011JA016482.

\section{Introduction}

[2] The United Nations (UN) Committee on the Peaceful Uses of Outer Space (COPUOS) defines space debris as "all man-made objects, including fragments and elements thereof, in Earth orbit or re-entering the atmosphere, that are non-functional" [United Nations Committee on the Peaceful Uses of Outer Space (UN COPUOS), 2010]. Space debris is found in numerous categories, from large, dead spacecraft and used launch vehicle upper stages, to centimeter-size solid rocket motor slag, and paint flakes and fragments in the micrometer size regime. Approximately 22,500 objects larger than $10 \mathrm{~cm}$ are known to exist, while the population of particles between 1 and $10 \mathrm{~cm}$ is estimated to be 500,000 and the number of smaller particles likely exceeds tens of millions (NASA Orbital Debris Program Office Frequently Asked Questions, http:// orbitaldebris.jsc.nasa.gov). The sources of this debris population are space launches, on-orbit operations and fragmentations, with the latter accounting for over half of the trackable objects in the current catalog.

\footnotetext{
${ }^{1}$ Astronautics Research Group, Faculty of Engineering and the Environment, University of Southampton, Southampton, UK.

Copyright 2011 by the American Geophysical Union. 0148-0227/11/2011JA016482
}

[3] Space debris is now widely known to represent a significant risk to operational spacecraft due to the collision hazard it represents. Indeed, the first collision between two intact objects in February 2009 led to the loss of Iridium 33 (as well as a defunct Russian satellite) and generated over 1900 fragments $10 \mathrm{~cm}$ or larger. Orbital speeds are such that even small particles carry sufficient kinetic energy to cause significant damage to or even catastrophic breakup of operational spacecraft. The Iridium 33-Cosmos 2251 event is only the most recent collision involving trackable orbiting objects. The European Space Agency's Database and Information System Characterizing Objects in Space (DISCOS) describes the occurrence of four other collisions since 1991, and D. Wright (unpublished data, 2009, available at http:// www.ucsusa.org) suggests that a further four may have taken place (Figure 1). As the population of debris continues to grow, the probability of further collisions will consequently increase. This will have a considerable impact upon satellite operations, because operational spacecraft will experience many close approaches with nonzero collision probability. At the time of the first recorded on-orbit collision in 1996, the Computation of Miss Between Orbits (COMBO) program operated by the United States Space Control Centre (SCC) identified over half a million close approaches of under $100 \mathrm{~km}$ between cataloged objects per day [Payne, 1997]. Although just a small fraction of close approaches ultimately result in a collision avoidance maneuver, routine 


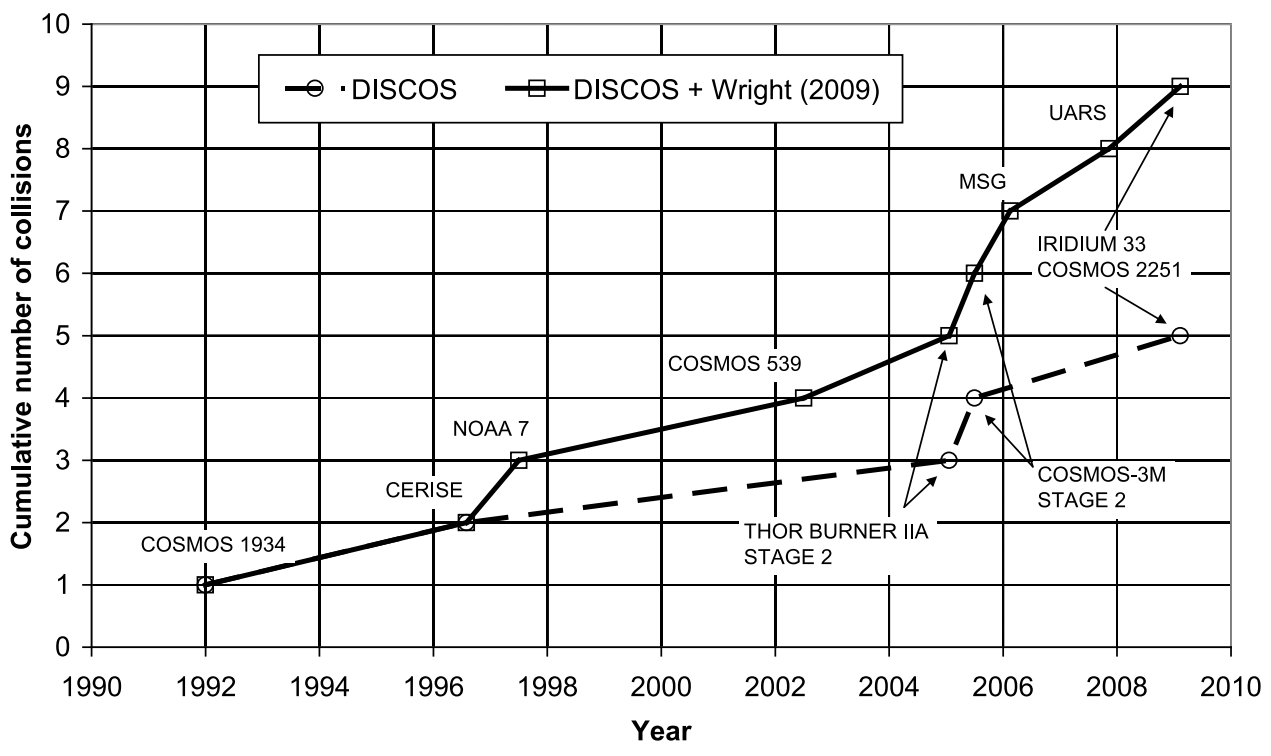

Figure 1. Historical collision activity.

conjunction assessment requires significant operational support at considerable cost.

[4] Atmospheric decay remains the only effective sink mechanism for space debris up to altitudes of about $600 \mathrm{~km}$. The drag acceleration, $a_{D}$, on a satellite with cross-sectional area $A$ and mass $M$ is a linear function of the local mass density, $\rho$,

$$
a_{D}=\frac{1}{2} \frac{A}{M} C_{D} \rho v_{r}^{2},
$$

where $C_{D}$ is the drag coefficient and $v_{r}$ is the velocity of the satellite relative to the atmosphere. A decrease in mass density will, thus, produce a corresponding decrease in the drag acceleration on a satellite, leading to an increase in the orbital lifetime. The orbit lifetime of an uncontrolled space vehicle is [Stark and Swinerd, 2003]

$$
\tau \approx \frac{e^{2}}{2 B}\left(1-\frac{11}{6} e+\frac{29}{16} e^{2}+\frac{7 H_{r_{p}}}{8 a}\right),
$$

where $e$ and $a$ are the eccentricity and semimajor axis after control has ceased, $H_{r_{p}}$ is the density scale height of the atmosphere at perigee, $r_{p}$, and $B$ is given by

$$
B \approx \sqrt{\left(\frac{\mu}{a^{3}}\right)} \frac{A C_{D}}{M} \rho_{r_{p}} a e I_{1}\left(\frac{a e}{H_{r_{p}}}\right) \exp \left(-e\left(1+\frac{a}{H_{r_{p}}}\right)\right) .
$$

Here, $I_{1}$ is the Bessel function of the first kind and order one and $\rho_{r_{p}}$ is the local mass density at the orbit perigee.

[5] Solar irradiance, especially at ultraviolet wavelengths, is a key driver of mass density change in the thermosphere, which expands and contracts as a result of temperature changes. Changes in solar irradiance over the 11 year solar cycle cause corresponding mass density changes of up to an order of magnitude [Emmert and Picone, 2010]. These changes in density cause large variations in atmospheric drag, from (1). Therefore, the rate at which orbiting objects re-enter the atmosphere during solar minima is typically less than the rate at which new objects are added to the environment, leading to a net increase in the LEO population for this part of the solar cycle. However, the effect is reversed during solar maxima as the thermospheric mass density increases and there is typically a net decrease in the population of objects in LEO over this period.

[6] Temperature changes in the mesosphere and lower thermosphere (60-100 km altitude) are also caused by the excitation of atmospheric $\mathrm{CO}_{2}$ by collisions with atomic oxygen, which result in infrared emission at $15 \mu \mathrm{m}$ and a net cooling [Akmaev and Formichev, 2000]. The anticipated doubling of the concentration of $\mathrm{CO}_{2}$ by the end of the $21 \mathrm{st}$ century [Brasseur and Hitchman, 1988] is expected to lead to a cooling of $10 \mathrm{~K}$ in the thermosphere, increasing to $50 \mathrm{~K}$ in the mesosphere [Roble and Dickinson, 1989], and a corresponding decrease in mass density at higher altitudes. It is likely that changes in mass density here would be due to contraction at lower altitudes, where the collision rates of $\mathrm{CO}_{2}$ and atomic oxygen are higher, and the subsequent collapse of the density structure above. In addition, the capacity of the stratospheric ozone layer to absorb solar ultraviolet radiation, which leads to the expansion of the atmosphere, has been compromised by the persistence of chlorofluorocarbons [Braathen et al., 2011]. As such, the depletion of stratospheric ozone may contribute to the net negative temperature trend at this altitude and the exacerbation of mass density changes in the thermosphere [Akmaev and Formichev, 2000].

[7] Empirical studies of thermospheric density change using satellite drag data have been performed by Keating et al. [2000], Emmert et al. [2004, 2008], and A. Saunders et al. (Further evidence of long-term thermospheric density change using a new method of satellite ballistic coefficient estimation, submitted to Journal of Geophysical Research, 2010). In general, these studies derive total mass densities, or changes in total mass densities, from a subset of the TwoLine Element (TLE) catalog generated by the U.S. Space 


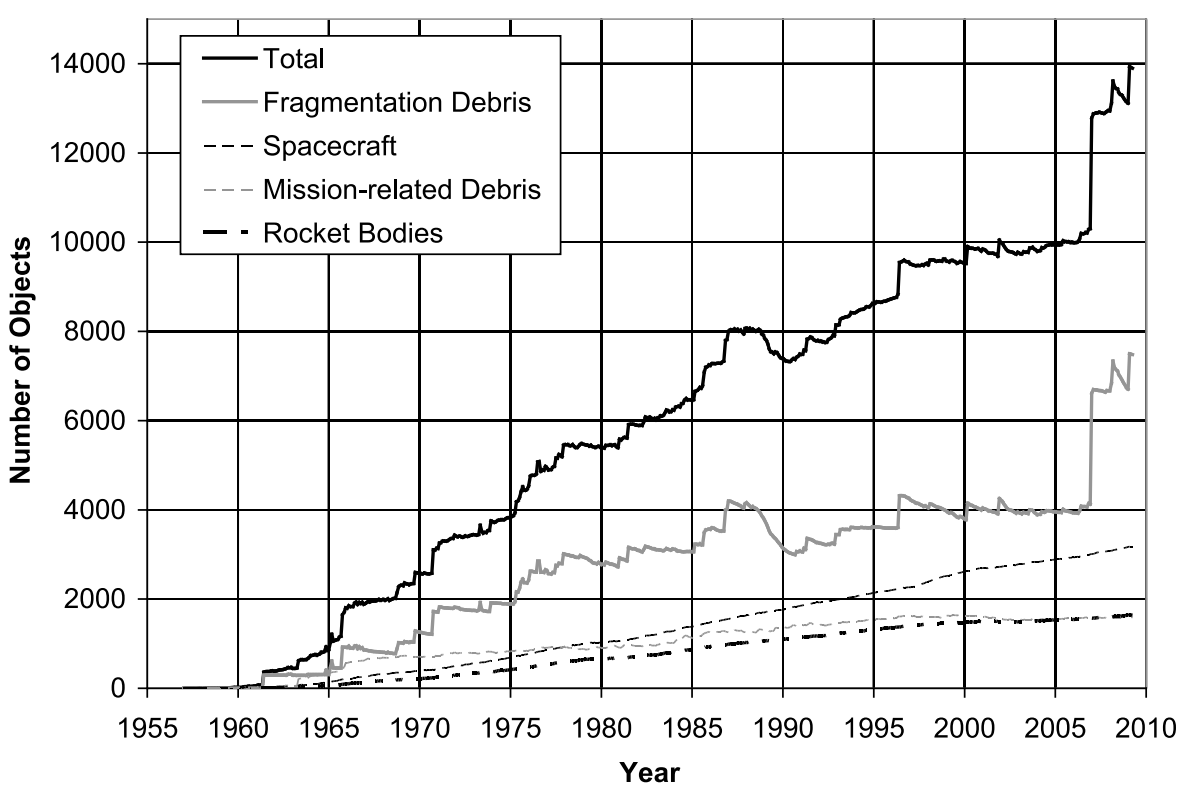

Figure 2. Monthly number of objects in Earth orbit (source: NASA).

Surveillance Network. Findings from these studies suggest an overall thermospheric density trend in the range $-2 \%$ to $-5 \%$ per decade. While the magnitude of this change is much smaller than the variations caused by the 11 year solar cycle, its secular nature could lead to a reduction in thermospheric density at a given height to half of its present value within 100 years [Emmert et al., 2004] if $\mathrm{CO}_{2}$ concentrations increase as expected. As before, the density reduction will lead to increasing lifetimes for satellites and space debris, and a corresponding decrease in the rate at which objects decay due to atmospheric drag. Simulations of the space debris environment have shown that these effects augment the collision rate and increase the number of objects $>10 \mathrm{~cm}$ above the level expected by increasing satellite lifetimes alone [Lewis et al., 2005].

[8] In 2007, the Inter-Agency Space Debris Coordination Committee (IADC), the intergovernmental forum created to discuss the technical issues associated with space debris, published a set of debris mitigation guidelines aimed at reducing these risks (available from http://www.iadc-online. org). In addition, the United Nations (UN) General Assembly adopted resolution 62/217 in December 2007, endorsing the Space Debris Mitigation Guidelines of UN COPUOS. These voluntary guidelines outline key measures for the planning, design, manufacture and operation of spacecraft and launch vehicles that are encapsulated within seven mitigation guidelines [UN COPUOS, 2010]: (1) limit debris released during normal operations; (2) minimize the potential for breakups during operational phases; (3) limit the probability of accidental collision in orbit; (4) avoid intentional destruction and other harmful activities; (5) minimize potential for postmission breakups resulting from stored energy; (6) limit the long-term presence of spacecraft and launch vehicle orbital stages in the low Earth orbit region after the end of their mission; and (7) limit the long-term interference of spacecraft and launch vehicle orbital stages with the geosynchronous region after the end of their mission.
[9] The qualitative, high-level UN COPUOS space debris mitigation guidelines follow the more detailed and technical IADC guidelines. Broadly speaking, the first five guidelines are aimed at preventing the generation of debris in the short term, whereas the last two guidelines focus on reducing debris generation in the long term by limiting the lifetime of defunct satellites and launch vehicle stages in key altitude regimes used by operational spacecraft.

[10] For spacecraft or orbital stages that end their mission in orbits that pass through the low Earth orbit (LEO) region, the IADC space debris mitigation guideline recommends direct atmospheric re-entry, retrieval or maneuver into an orbit with a lifetime of 25 years (IADC, 2007). For some LEO spacecraft and orbital stages, an alternative is to maneuver into a storage orbit with a perigee altitude above $2000 \mathrm{~km}$. In most cases, however, the solution preferred by satellite operators is to maneuver into a 25 year, lifetimelimited orbit as this involves the lowest cost. Drag augmentation devices may also be used to reduce the orbit lifetime, although such devices should reduce the area-time product, $A \tau$, of the vehicle, or demonstrate that an impact with another spacecraft or large debris will not cause further fragmentation (U.S. Government Orbital Debris Mitigation Standard Practices, available at http://www.orbitaldebris.jsc. nasa.gov). The perigee altitude for which

$$
\tau-25 \text { years }=0
$$

identifies an orbit that satisfies the 25 year orbit lifetime and, thus, meets the IADC debris mitigation guideline. The root of the nonlinear equation (4) can be determined using the bisection method, thereby guaranteeing convergence.

[11] The adoption of space debris mitigation guidelines has succeeded in reducing the growth in the population of launch vehicle stages and mission-related debris since the beginning of the 21st century (Figure 2). However, computer simulations conducted in the last four years have 
suggested that the current debris population in LEO has reached a sufficient density at some altitudes for collision activity there to continue even in the absence of new launches [Liou and Johnson, 2006]. Further, the rate at which new debris is generated by these collisions exceeds the rate at which it is removed by atmospheric decay, leading to a net growth of the space debris population in LEO. In reality, the situation will be worse than this 'no new launches' scenario: spacecraft will continue to be launched and major, unexpected breakups will continue to occur. Debris mitigation practices, such as those described above, will help to limit the rate of growth but will be insufficient. The removal of large, intact objects from critical altitudes, where high levels of collision activity are expected, is now being considered to stabilize the population growth [Liou and Johnson, 2007a].

[12] Simulations of active debris removal (ADR) have demonstrated, in principle, that the LEO debris population can be stabilized by the removal of a relatively few, selected debris targets [Lewis et al., 2009; Liou and Johnson, 2007b]. According to these simulations, prevention of the growth of the existing LEO population can be achieved by five removals per year, beginning in the year 2020. On average, for every object removed through ADR the total debris population is reduced by 10 [Lewis et al., 2009]. Lower removal rates have been shown to be less effective and may not be sufficient to constrain the growth of the LEO debris population. It is likely that a negative secular density trend will result in a similar reduction in the effectiveness of ADR.

[13] In this paper, we employ the University of Southampton's Debris Analysis and Monitoring Architecture for the Geosynchronous Environment (DAMAGE) to quantify the impact of the secular decline in thermospheric density on the effectiveness of debris mitigation and removal practices.

\section{The DAMAGE Model}

[14] The University of Southampton's debris model is a three-dimensional computational model that was initially developed to simulate the debris population in geosynchronous Earth orbit (GEO) but has since been upgraded to allow investigations of the full LEO to GEO debris environment. DAMAGE is a semideterministic model implemented in $\mathrm{C}++$, running under Microsoft Windows and uses OpenGL for graphical support. A fast, pair-wise algorithm based on the 'Cube' approach adopted in NASA's LEO-to-GEO Environment Debris model (LEGEND) [Liou et al., 2004] is used to determine the collision probability for all orbiting objects. The collision probability of an object $i$ with a second object $j$ in a small cubic volume element $d U$ over a short time interval $d t$ can be expressed as [Liou, 2006]

$$
d P_{i, j}(t)=s_{i} s_{j} v_{i j} \sigma d U d t
$$

where $s_{i}$ and $s_{j}$ are the residential probabilities (also spatial densities) of objects $i$ and $j$ in the cube $d U, v_{i j}$ is the velocity of object $j$ relative to object $i$, and $\sigma$ is the combined crosssectional area of both objects measured in a plane normal to the relative velocity. The integration of (5) for all objects $j \neq i$ over a relatively long projection period (e.g., decades or centuries), and over the volume of near-Earth space provides an estimate of the cumulative collision probability, $P_{i, j}(t)$, for objects $i$ and $j$. In practice, $d P_{i, j}(t)$ is calculated at discrete time intervals only for cases where two objects occupy the same cubic volume element. Thus, the computation time increases with $N$ rather than $N^{2}$ for an environment containing $N$ objects and sampling is performed over time so that new objects and changing orbital elements are incorporated [Liou, 2006]. A uniformly distributed random number is generated and compared with $d P_{i, j}(t)$ to determine whether a collision between objects $i$ and $j$ at time $t$ actually occurs. If so, DAMAGE makes use of the NASA Standard breakup model [Johnson et al., 2001] to generate fragmentation debris.

[15] DAMAGE employs a fast, semianalytical orbital propagator to update the orbital elements of objects within the environment. This propagator includes orbital perturbations due to Earth gravity harmonics, $J_{2}, J_{3}$, and $J_{2,2}$, lunisolar gravitational perturbations, solar radiation pressure and atmospheric drag. The drag model assumes a nonrotating, oblate atmosphere with density and density scale height values taken from the 1972 COSPAR International Reference Atmosphere (CIRA). This model was chosen for its relative simplicity, as computational efficiency is important for evolving large populations over long time periods. Atmospheric density and scale height values are stored as look-up tables within DAMAGE for discrete altitudes and exospheric temperatures. The exospheric temperature, $T_{e x}(t)$, at time $t$ is given by

$$
T_{e x}(t)=1.15\left(379+\left[3.24 F_{10.7}(t)\right]\right),
$$

where $F_{10.7}(t)$ is the solar flux at a wavelength of $10.7 \mathrm{~cm}$. Projected solar activity is described in DAMAGE using the sinusoidal model in Figure 3. Log linear interpolation is used to extract density and scale height estimates from the look-up tables at the perigees of all objects within the LEO region for $F_{10.7}(t)$ values throughout the projection period.

[16] Following the approach adopted by Lewis et al. [2005], DAMAGE accounts for thermospheric cooling using an empirically derived secular density change. In the 2005 work, results from the analysis of 27 long-lived satellites reported by Emmert et al. [2004] were used to develop a simple model of the density trend, $\Delta \rho$,

$$
\Delta \rho=-3.4+0.01441\left(F_{10.7}(t)-70\right)-0.0036(h-240),
$$

as a percentage per decade and as a function of the $F_{10.7} \mathrm{~cm}$ solar flux and height above sea level, $h$. In this paper, we use the Earth orbital propagator for thermospheric analysis (AETHER) (Saunders et al., submitted manuscript, 2010) to predict the thermospheric density changes using Two-Line Element (TLE) sets. AETHER employs models of Earth gravity harmonics to order and degree 20, and atmospheric drag is predicted using the empirical atmospheric density model Naval Research Laboratory's Mass Spectrometry and Incoherent Scatter Radar up to the Exobase, released in the year 2000 (NRLMSISE-00). Other orbital perturbations included in AETHER are the third-body gravitational effects due to the Moon and Sun, and solar radiation pressure with an oblate, conical Earth shadow. The propagator and its use to derive density trends from 41 LEO satellites are described by Saunders et al. For a selected, long-lived sat- 


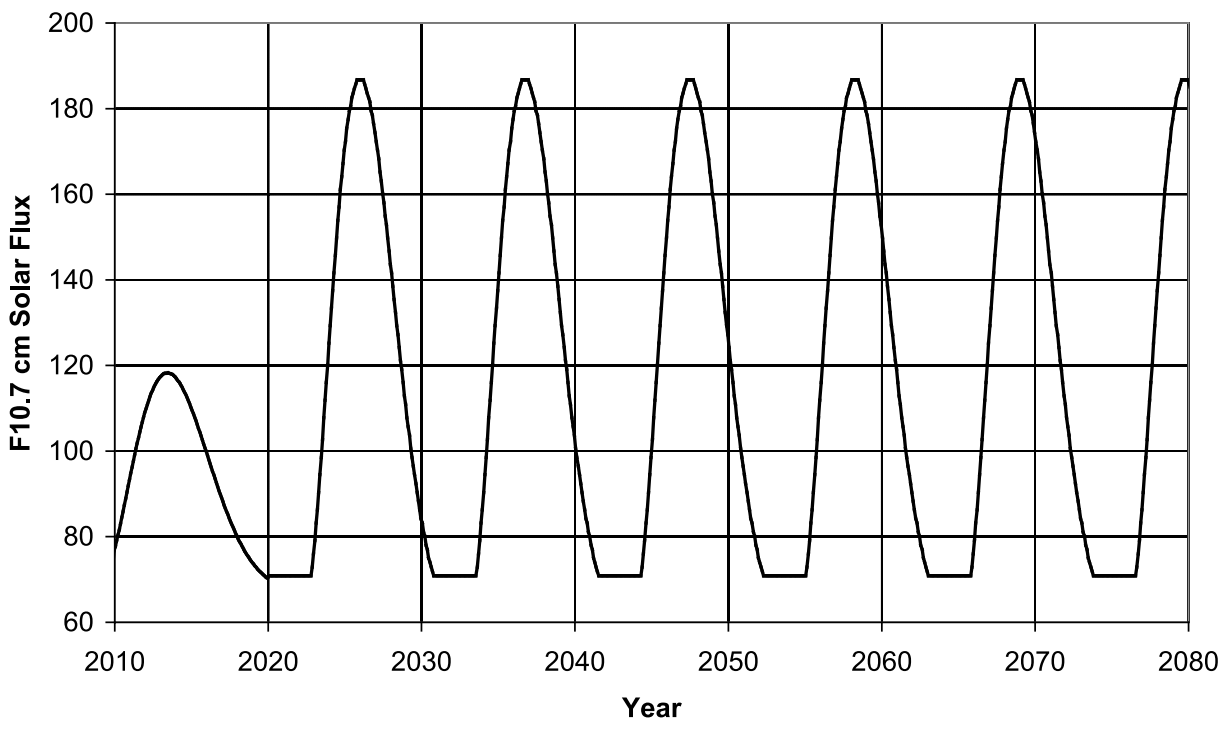

Figure 3. $F_{10.7} \mathrm{~cm}$ solar flux projection used in DAMAGE.

ellite, the ratio, $\zeta$, of the 'observed' thermospheric mass density, $\rho$, derived using TLE sets, to the 'predicted' value, $\rho^{*}$, derived using AETHER, is

$$
\zeta=\frac{\rho}{\rho^{*}}
$$

and characterizes the density trend. This ratio is approximated by,

$$
\zeta=\frac{\Delta a}{\Delta a^{*}}
$$

where $\Delta a$ is the difference in the semimajor axis, $a$, of the satellite from one TLE set to some later TLE set, and $\Delta a^{*}$ is the difference in semimajor axis computed by AETHER over the same period of time. The density ratio is associated with a particular altitude depending on the size of the semimajor axis, $a$, the orbit eccentricity, $e$, and the density scale height of the atmosphere at the orbit perigee [KingHele, 1987]. Overall, this approach differs from the method described by Picone et al. [2005] and employed by Emmert et al. [2004, 2008, 2010] in that (9) provides an estimate of the relative change in total mass density, rather than being a prediction of the total mass density itself.

[17] Here, AETHER was used to infer thermospheric density values from four decades of TLE data for $30 \mathrm{sa}-$ tellites in LEO, including data from the record low density of the 2008 solar minimum. From these results, an empirical model of the density trend was developed; the modified density (incorporating thermospheric contraction),

$$
\rho_{c}=\rho_{i}\left\lfloor(0.98028-0.00013 h)^{T}\left(0.00109 F_{10.7}(t)+0.88578\right)\right\rfloor,
$$

at height $h$ and for $10.7 \mathrm{~cm}$ solar flux, $F_{10.7}(t)$, is estimated from the initial density, $\rho_{i}$, obtained using log linear interpolation of CIRA-72 density values stored in the DAMAGE look-up table, and at time $T$ decades measured from the epoch 1 January 1970. For $h=300 \mathrm{~km}$, the cooling trend given by (10) is shown in Figure 4.

[18] Using satellite drag data, Emmert et al. [2010] were able to describe record low thermospheric mass densities during the prolonged 2008 solar minimum that were 10 $30 \%$ lower than predicted by NRLMSISE-00, due, in part, to the radiative cooling effects of atmospheric $\mathrm{CO}_{2}$. As the empirical model in (10) included data from this period, it is likely that mass density changes it forecasts are biased toward large, negative values. Potentially, this bias results in overestimates of spacecraft and debris lifetimes, especially toward the end of the environment projection period.

[19] Debris environment projections covering the period from October 1957 to July 2009 (inclusive) and employing historical launch and fragmentation information from ESA's DISCOS have been used to establish LEO populations for the epoch 1 August 2009. Two versions of this population were generated; one using unmodified CIRA-72 densities and the second employing (10) from 1 January 1970. Sub-

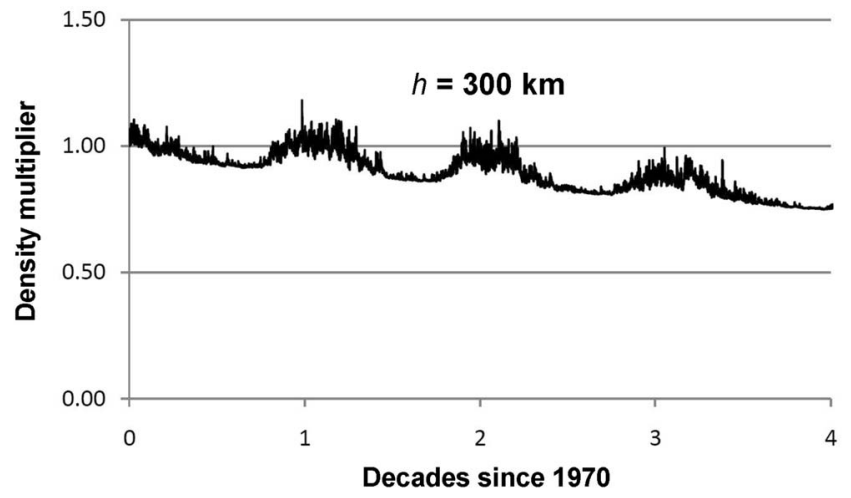

Figure 4. Density trend at a height of $300 \mathrm{~km}$ from epoch 1 January 1970. 
Table 1. Description of the Benchmark Mitigation Scenario

\begin{tabular}{ll}
\hline \multicolumn{1}{c}{ Parameter } & Value \\
\hline $\begin{array}{l}\text { Projection period } \\
\text { Traffic model (2009-2079) }\end{array}$ & $\begin{array}{c}\text { 1 August 2009-1 August 2079 } \\
\text { Repeat 10 year (1999-2009) launch } \\
\text { traffic } \\
\text { Move spacecraft and rocket bodies } \\
\text { to 25 year decay orbits } \\
\text { Postmission disposal } \\
(2009-2079)\end{array}$ \\
$\begin{array}{l}\text { No explosions } \\
\text { (2009-2079) }\end{array}$ & 5 days \\
Time step & $10 \mathrm{~cm}$ \\
$\begin{array}{l}\text { Minimum object size } \\
\text { Collision prediction: } \\
\text { cube size }\end{array}$ & $10 \mathrm{~km}$ \\
\hline
\end{tabular}

sequent projections into the future of the debris population $\geq 10 \mathrm{~cm}$ commence from this epoch and are performed using a Monte Carlo (MC) approach to account for stochastic elements within the model and to establish reliable statistics. Future projections repeat launch traffic from 1999 to 2009 on a 10 year cycle. In addition, the density trends predicted by (10) were assumed to continue unchanged throughout the projection period.

\section{Method}

[20] A 70 year future projection from 1 August 2009 was used by DAMAGE as the benchmark scenario for this investigation (Table 1). This scenario incorporated key elements of the UN COPUOS and IADC space debris mitigation guidelines, including postmission disposal (PMD) to limit the lifetime of spacecraft and launch vehicle orbital stages in the LEO region. These objects were moved to 25 year decay orbits or LEO storage orbits (above LEO) depending on the $\Delta v$. As the storage orbits were above LEO, the region of interest to this study, objects placed here were no longer processed in the simulation. PMD measures were implemented from the start of the future projection and were applied to $90 \%$ of all eligible objects. In addition to the PMD measures, it was assumed that no explosions occurred during operational phases, and all spacecraft and upper stages were passivated at end of mission. That is, no explosive breakups occurred in the projection period.

[21] The benchmark mitigation scenario was enhanced in a second series of simulations using a simple ADR strategy. Intuitively, it makes sense to target objects for ADR based on their contribution to future collision activities. Thus, the probability of an object being involved in a collision, and the number of fragments added to the environment if a collision does occur, have been factors used to define criteria for ADR in previous studies. For example, Liou and Johnson [2007b] used the criterion

$$
R_{i}(t)=m_{i} \sum_{j \neq i} P_{i, j}(t)
$$

to rank objects for removal, where $m_{i}$ is the mass of object $i$, which is a key factor in the NASA standard breakup model for determining the number of collision-induced fragments [Johnson et al., 2001]. The estimation of the cumulative probability $\sum_{j \neq i} P_{i, j}(t)$ over all objects $j \neq i$ for use in this removal criterion is made outside the normal environment projection and is achieved using an integration of (5) over a relatively short time interval (days), at the start of each ADR year [Liou et al., 2004]. However, this process can be timeconsuming and a number of alternative criteria are available.

[22] For this paper, we utilized the results of a previous DAMAGE study to identify potential ADR targets [Lewis et al., 2011]. For each of $100 \mathrm{MC}$ runs of this scenario, DAMAGE recorded the altitude of all collisions (Figure 5). This is the integration over time of (5) but the purpose of this particular integration is to identify a critical altitude

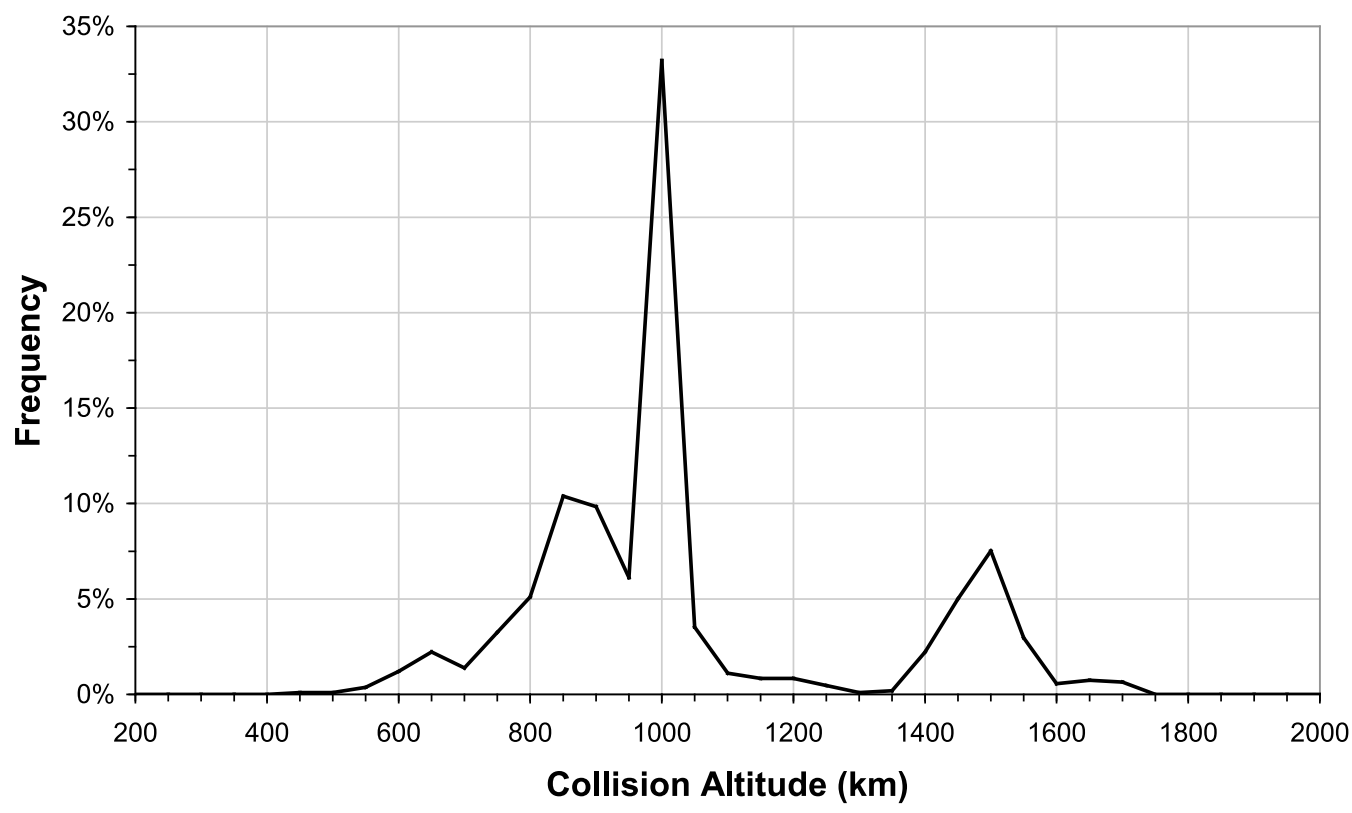

Figure 5. Collision frequency as a function of altitude, determined from the DAMAGE benchmark mitigation study. 
Table 2. Description of the DAMAGE Mitigation and ADR Scenarios

\begin{tabular}{lc}
\hline \multicolumn{1}{c}{ Scenario } & Description \\
\hline $\begin{array}{l}\text { Mitigation no contraction } \\
\text { Mitigation with contraction }\end{array}$ & As in Table 1, using unmodified CIRA-72 densities \\
ADR no contraction & As in Table 1, using modified CIRA-72 densities from (10) \\
& As in Table 1, five most massive objects in 950-1050 km band \\
ADR with contraction & removed immediately per year (on 1 January) from 2020 \\
& As in Table 1, five most massive objects in 950-1050 km band \\
& removed immediately per year (on 1 January) from 2020. \\
& Modified CIRA-72 densities from (10) \\
\hline
\end{tabular}

band as the focus of ADR rather than particular spacecraft or launch vehicle stages. The expectation from the results of this previous study were that collisions in the altitude band $950 \mathrm{~km}$ to $1050 \mathrm{~km}$ are three times more likely than at other altitudes. Following previous ADR studies, which have identified an effective removal rate, the DAMAGE ADR scenario in this paper targeted the five most massive objects per year from the $950-1050 \mathrm{~km}$ altitude band, beginning 1 January 2020. In addition, the following eligibility requirements for removal were used.

[23] 1. The object must be intact (i.e., a payload, launch vehicle upper stage or mission-related debris).

[24] 2. The object must have an orbital eccentricity $<0.5$.

[25] 3. The object must have a perigee altitude $<1400 \mathrm{~km}$.

[26] 4. The object must not already be subject to PMD measures.

[27] Two versions of the mitigation and ADR scenarios were used to investigate the effects of thermospheric contraction on the future LEO space debris population. In the first version, unmodified CIRA-72 densities were used to estimate atmospheric drag (and PMD decay orbit perigees) whereas the second version utilized CIRA-72 densities modified according to (10). These two mitigation and two ADR scenarios are described in Table 2 along with a third ADR scenario used for comparison purposes.

[28] An effective reduction factor (ERF), introduced by Liou and Johnson [2007a], was calculated to quantify the effectiveness of the ADR scenarios investigated in this study, where

$$
E R F(t)=\frac{N(t)-N_{S}(t)}{C N_{R}(t)}
$$

$N(t)$ is the effective number of objects $\geq 10 \mathrm{~cm}$ in the benchmark mitigation (no ADR) scenario at time $t, N_{S}(t)$ is the effective number of objects $\geq 10 \mathrm{~cm}$ in the ADR scenario at time $t$, and $C N_{R}(t)$ is the cumulative number of objects removed at time $t$. The effective number is defined as the fractional time, per orbital period, an object spends in LEO. The ERF quantifies the reduction in the total population for each object removed through $\operatorname{ADR} . E R F(t)$ is a function of time and can thus be calculated at any point in the projection period. Liou and Johnson [2007a] report ERF values calculated at the end of the projection period. However, the mean ERF calculated from the year 2021 to the end of the projection period provides a more robust measure [Lewis et al., 2009].

\section{Results and Discussion}

[29] The effective number of objects in LEO over the projection period for the two mitigation and the two ADR scenarios are shown in Figure 6. Only the averages of the $50 \mathrm{MC}$ runs are shown, for clarity. For all scenarios, there is

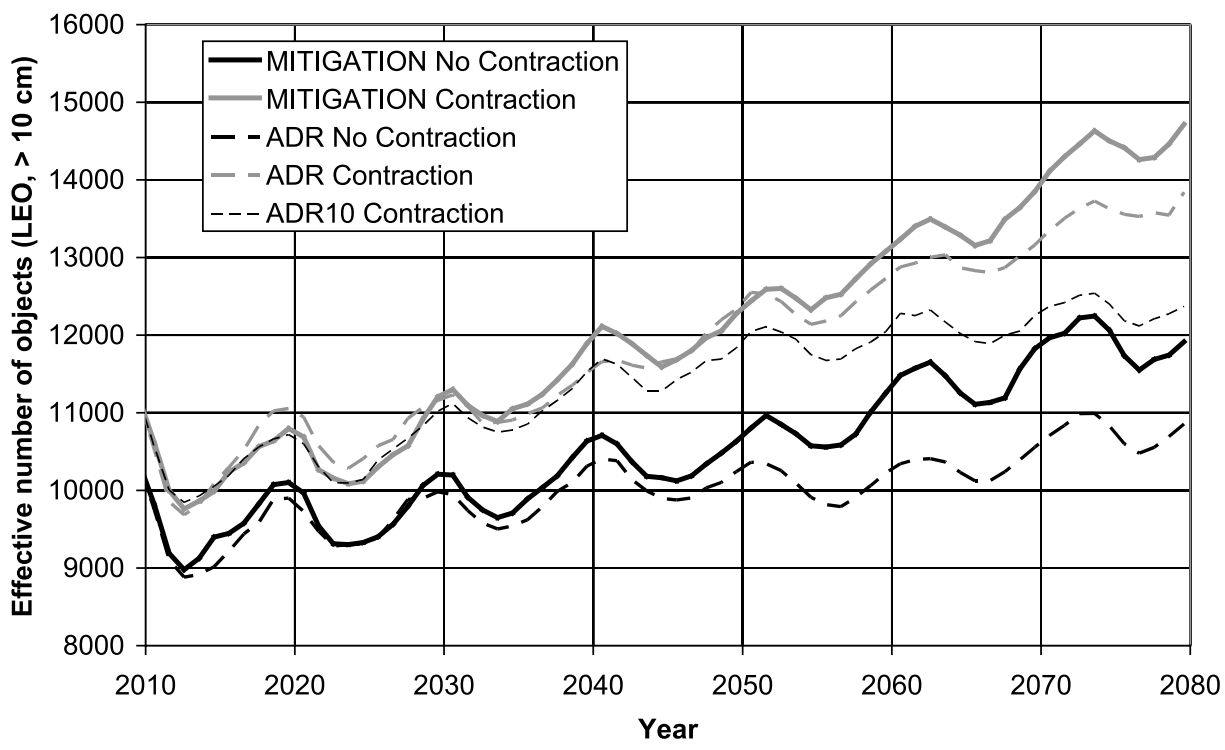

Figure 6. Projected number of objects in LEO for DAMAGE mitigation and ADR scenarios. 


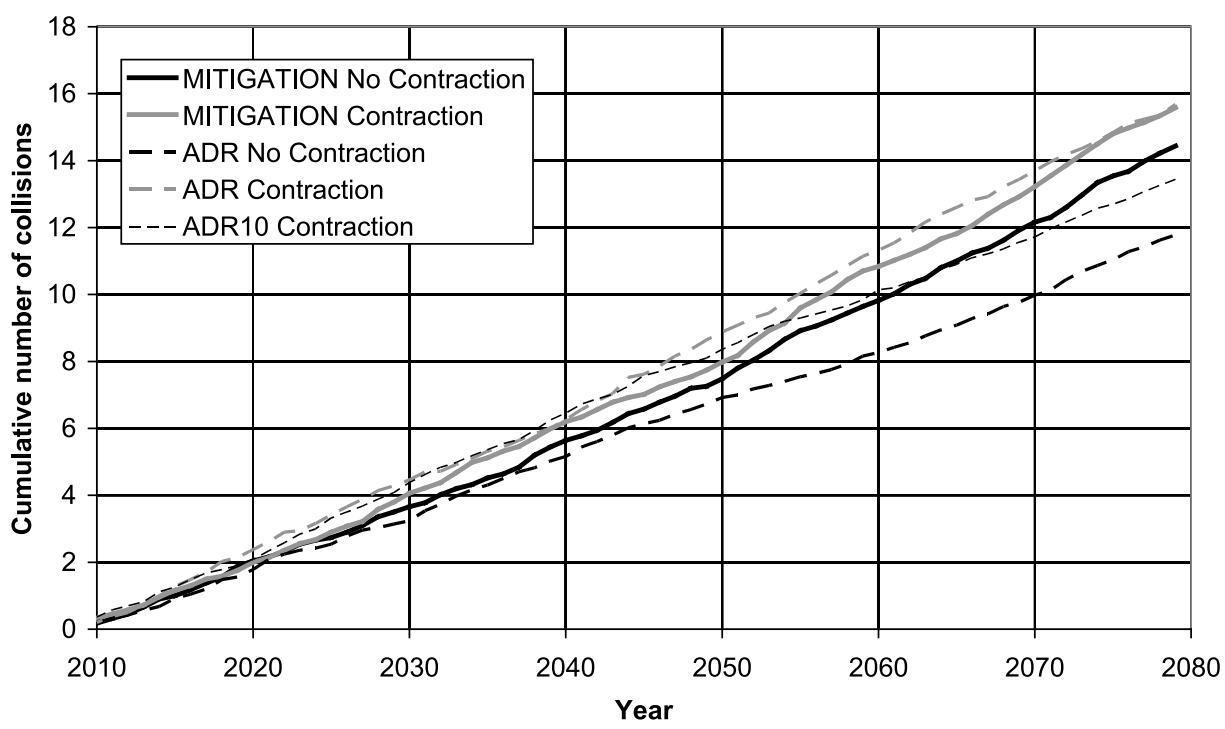

Figure 7. Projected number of collisions in LEO for DAMAGE mitigation and ADR scenarios.

a rise in the population as a result of sustained collision activity throughout the projection period (Figure 7). For the benchmark mitigation scenario without thermospheric contraction, the mean LEO population increase was 1568 $(1$-sigma $=1908)$ objects from 10,346 at the 1 August 2009 epoch (Table 3). When ADR was employed, at a rate of five removals per year from 1 January 2020, the population grew by a mean of $508(1$-sigma $=1551)$ objects. With the expectation that the population in the mitigation-only scenario would be larger than that in the ADR scenario, the observed difference in the size of the population does reach significance in a one-tailed test (with coefficient of determination, $F_{(1,49)}=1.514$, and probability, $p=0.075$, where $F_{(1,49)}$ denotes a Fisher-Snedecor distribution with 1 degree of freedom in the numerator and 49 degrees of freedom in the denominator [Moore et al., 2009]). ADR reduced the LEO population by 1060 objects by the end of the projection $(E R F(t)=3.5, t=1$ Aug. 2079), compared with mitigation only (Table 3 ). For each object removed by ADR the mean population reduction is $4.1(1$-sigma $=6.1)$.

[30] When thermospheric contraction was included in the mitigation-only simulation, the mean LEO population increase was $3543(1$-sigma $=2461)$ from 11,173 on 1 August 2009. Statistical analysis shows the effect of thermospheric contraction to be significant for the mitigation- only scenarios $\left(F_{(1,49)}=0.397, p=0.008\right)$. With ADR and thermospheric contraction, the mean population increase was $2669(1$-sigma $=2010)$ objects. While this represents a reduction of 874 objects by 1 August 2079 compared with the mitigation-only scenario $(E R F(t)=2.9, t=1$ Aug. 2079) and is significant in a one-tailed test $\left(F_{(1,49)}=1.499, p=\right.$ $0.080)$, the mean ERF value is $-0.9(1$-sigma $=8.6)$. Inspection of $E R F(t)$ values over the projection period (Figure 8) reveals that $E R F(t) \leq 1$ in the periods 2020 through 2034 and 2044 through 2053. This is in contrast to the ADR scenario without thermospheric contraction for which $\operatorname{ERF}(t) \geq 1$ from 2028 onwards.

[31] The yearly increases in the LEO populations from all scenarios were estimated by fitting linear trend lines to the curves in Figure 6. The yearly collision rates were found using a similar procedure. The results of this fitting process and corresponding correlation coefficient $\left(R^{2}\right)$ values are shown in Table 4. For the benchmark mitigation scenario (without thermospheric contraction), the LEO population grew by 39 objects per year $\left(R^{2}=0.851\right)$ and the collision rate was 0.21 per year $\left(R^{2}=0.996\right)$. When ADR was employed, with five removals per year, the LEO population growth rate was halved, to 19.6 objects per year $\left(R^{2}=\right.$ 0.678 ), and the collision rate decreased to 0.17 events per year $\left(R^{2}=0.999\right)$. However, thermospheric contraction

Table 3. Number of Objects in LEO and ADR Effectiveness

\begin{tabular}{|c|c|c|c|c|c|}
\hline Scenario & $\begin{array}{c}\text { Number of } \\
\text { Objects } \\
\text { (1 August 2009) }\end{array}$ & $\begin{array}{l}\text { Mean Number } \\
\text { of Objects } \\
\text { (1 August 2079) }\end{array}$ & $\begin{array}{l}\text { Mean Number } \\
\text { Reduced } \\
\text { cf. Mitigation } \\
\text { (1 August 2079) }\end{array}$ & $\begin{array}{c}\text { ERF } \\
\text { (1 August 2079) }\end{array}$ & Mean ERF \\
\hline Mitigation no contraction & 10,346 & 11,914 & - & - & - \\
\hline Mitigation contraction & 11,173 & 14,716 & - & - & - \\
\hline ADR no contraction & 10,346 & 10,854 & 1060 & 3.5 & 4.1 \\
\hline ADR contraction & 11,173 & 13,842 & 874 & 2.9 & -0.9 \\
\hline ADR10 contraction & 11,173 & 12,369 & 2347 & 3.9 & 2.0 \\
\hline
\end{tabular}




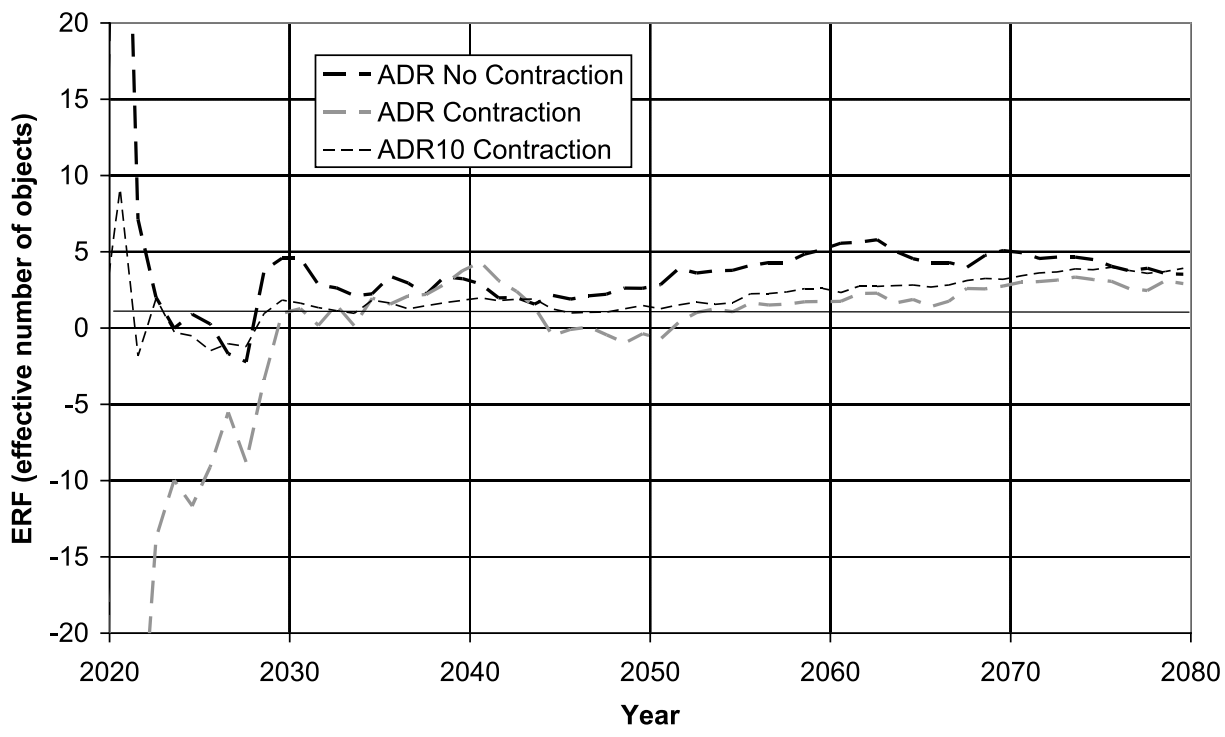

Figure 8. Effective reduction factor for DAMAGE ADR scenarios. The solid line indicates the ERF level above which ADR is effective.

reduces the effectiveness of ADR, such that the LEO population growth rate falls from 67.9 objects per year without $\operatorname{ADR}\left(R^{2}=0.942\right)$ to 53.6 objects per year with $\mathrm{ADR}\left(R^{2}=\right.$ $0.937)$.

[32] The density trend increased the LEO population growth rate by $74 \%$ in the mitigation-only scenario and by $173 \%$ in the combined mitigation-ADR scenario. Thus, the effect of the thermospheric contraction appears greater for scenarios incorporating ADR although a different removal criterion (e.g., (11)) may lessen its impact. Another way to restore the benefits of ADR is to increase the yearly removal rate. Results from the final DAMAGE simulation ("ADR10 Contraction") showed that if the ADR rate was doubled, to 10 removals per year, the LEO population growth rate was decreased to 35.2 objects per year (Table 4). Ten removals per year also cut the collision rate by nearly $14 \%$. The LEO population still grew by a mean of $1196 \pm 1881$ objects but was reduced by 2347 objects by 1 August 2079 compared with the benchmark mitigation scenario with thermospheric contraction $(E R F(t)=3.9, t=1$ Aug. 2079; Table 3). A onetailed test showed this to be a significant result $\left(F_{(1,49)}=\right.$ $1.712, p=0.031)$. The mean $E R F(t)$ over the projection period was $2.0 \pm 1.6$. That is, for every object removed through ADR, the LEO population was reduced by 2.0 objects, on average.

\section{Conclusions}

[33] The LEO space debris population is expected to increase due to random collisions between existing on-orbit debris, even in the absence of new launch activities. The widespread adoption of debris mitigation measures will limit this growth but will be insufficient to stabilize the growth of the population. Remediation of the environment through an active debris removal campaign, whereby intact objects are removed, presents a possible solution to this problem and previous work has shown that removing relatively few objects per year can prevent the spontaneous growth of the existing LEO population. However, a secular decline in thermospheric density will decrease the effectiveness of key debris mitigation measures and ADR, as it directly affects the atmosphere as the primary sink mechanism for space debris. Work by Emmert et al. [2008], among others, has provided evidence for such a thermospheric contraction.

[34] In this paper, we have used AETHER to derive an empirical density trend, as a function of height and $F_{10.7} \mathrm{~cm}$ solar flux, and then assumed that this trend continues into the future. DAMAGE projections of the space debris population in LEO have shown that the density trend causes a significant reduction in the effectiveness of space debris mitigation measures and in the ability of a key remediation measure, ADR, to constrain the LEO population growth. The decline in thermospheric density results in a $74 \%$ increase in the LEO population growth rate over a 70 year projection of a mitigation-only scenario, and a $173 \%$ increase in the population growth rate for a combined mitigation-ADR scenario. The positive effects of ADR on the LEO debris population are effectively canceled by the density trend, as measured by the mean ERF value. Doubling the ADR removal rate from five objects per year to 10 objects per year restored the benefits of remediation in the simulations but doing this in practice would involve higher costs and operational complexity.

Table 4. Slope Values of Straight Line Fits to Curves in Figures 6 and $7^{\mathrm{a}}$

\begin{tabular}{lcc}
\hline \multicolumn{1}{c}{ Scenario } & $\begin{array}{c}\text { Number of } \\
\text { Objects/Year }\end{array}$ & $\begin{array}{c}\text { Number of } \\
\text { Collisions/Year }\end{array}$ \\
\hline Mitigation no contraction & $39.0(0.851)$ & $0.21(0.996)$ \\
Mitigation contraction & $67.9(0.942)$ & $0.22(0.995)$ \\
ADR no contraction & $19.6(0.678)$ & $0.17(0.999)$ \\
ADR contraction & $53.6(0.937)$ & $0.23(0.998)$ \\
ADR10 contraction & $35.2(0.872)$ & $0.19(0.998)$ \\
\hline
\end{tabular}

${ }^{\mathrm{a}}$ Correlation coefficient $\left(R^{2}\right)$ values are shown in parentheses. 
[35] Work, to establish the sensitivity of the results to changes in the ADR removal criteria and to incorporate models of drag augmentation devices into the simulation, is ongoing.

[36] Acknowledgments. Financial support for this work was provided by the Faculty of Engineering and the Environment, University of Southampton. The authors gratefully acknowledge the assistance provided by Sarah Stevenage (University of Southampton) with the statistical analysis.

[37] Robert Lysak thanks the reviewers for their assistance in evaluating this paper.

\section{References}

Akmaev, R. A., and V. I. Formichev (2000), A model estimate of cooling in the mesosphere and lower thermosphere due to the CO2 increase over the last 3-4 decades, J. Geophys. Res., 27(14), 2113-2116.

Braathen, G., et al. (2011), Observations of the Antarctic ozone hole from 2003 to 2010, paper presented at European Geosciences Union meeting, Vienna, April.

Brasseur, G., and M. Hitchman (1988), Stratospheric response to trace gas perturbations: Changes in ozone and temperature distributions, Science, 240, 634-637, doi:10.1126/science.240.4852.634.

Emmert, J. T., and J. M. Picone (2010), Climatology of globally averaged thermospheric mass density, J. Geophys. Res., 115, A09326, doi:10.1029/2010JA015298.

Emmert, J. T., J. M. Picone, J. L. Lean, and S. H. Knowles (2004), Global change in the thermosphere: Compelling evidence of a secular decrease in density, J. Geophys. Res., 109, A02301, doi:10.1029/2003JA010176.

Emmert, J. T., J. M. Picone, J. L. Lean, and R. R. Meier (2008), Thermospheric global average trends, 1967-2007, derived from orbits of 5000 near-Earth objects, Geophys. Res. Lett., 35, L05101, doi:10.1029/ 2007GL032809.

Emmert, J. T., J. L. Lean, and J. M. Picone (2010), Record low thermospheric density during the 2008 solar minimum, Geophys. Res. Lett., 37, L12102, doi:10.1029/2010GL043671.

Johnson, N. L., P. H. Krisko, J.-C. Liou, and P. D. Anz-Meador (2001) NASA's new breakup model of EVOLVE 4.0, Adv. Space Res., 28 1377-1384, doi:10.1016/S0273-1177(01)00423-9.

Keating, G. M., R. H. Tolson, and M. S. Bradford (2000), Evidence of long-term global decline in the Earth's thermospheric densities apparently related to anthropogenic effects, Geophys. Res. Lett., 27, 1523-1526, doi:10.1029/2000GL003771.

King-Hele, D. (1987), Satellite Orbits in an Atmosphere, Blackie Acad. and Prof., Glasgow, U. K.
Lewis, H. G., G. G. Swinerd, C. S. Ellis, and C. E. Martin (2005) Response of the space debris environment to greenhouse cooling, in Proceedings of the Fourth European Conference on Space Debris, Darmstadt, Germany, 18-20 April, 2005, Eur. Space Agency Spec. Publ., ESA SP-587, 243-248.

Lewis, H. G., G. G. Swinerd, R. J. Newland, and A. Saunders (2009), Active removal study for on-orbit debris using DAMAGE, in Proceedings of the Fifth European Conference on Space Debris, Darmstadt, Germany, 30 March-2 April 2009 [CD-ROM], Eur. Space Agency Spec. Publ., ESA SP-672.

Lewis, H. G., G. G. Swinerd, and R. J. Newland (2011), The space debris environment: Future evolution, Aeronaut. J., in press.

Liou, J.-C. (2006), Collision activities in the future orbital debris environment, Adv. Space Res., 38, 2102-2106, doi:10.1016/j.asr.2005.06.021.

Liou, J.-C., and N. L. Johnson (2006), Risks in space from orbiting debris, Science, 311, 340-341, doi:10.1126/science.1121337.

Liou, J.-C., and N. L. Johnson (2007a), Instability of the present LEO satellite populations, Adv. Space Res., 41, 1046-1053.

Liou, J.-C., and N. L. Johnson (2007b), A sensitivity study of the effectiveness of active debris removal in LEO, paper presented at 58th International Astronautical Congress, Int. Astronaut. Fed., Hyderabad, India.

Liou, J.-C., D. T. Hall, P. H. Krisko, and J. N. Opiela (2004), LEGENDA three-dimensional LEO-to-GEO debris evolutionary model, $A d v$. Space Res., 34, 981-986, doi:10.1016/j.asr.2003.02.027.

Moore, D. S., G. P. McCabe, and B. Craig (2009), Introduction to the Practice of Statistics, 6th ed., W. H. Freeman, New York.

Payne, T. P. (1997), First "confirmed" natural collision between two catalogued satellites, in Proceedings of the Second European Conference on Space Debris, Darmstadt, Germany, March 17-19 1997, Eur. Space Agency Spec. Publ., ESA SP-393, 597-600.

Picone, J. M., J. T. Emmert, and J. L. Lean (2005), Thermospheric densities derived from spacecraft orbits: Accurate processing of two-line element sets, J. Geophys. Res., 110, A03301, doi:10.1029/2004JA010585.

Roble, R. G., and R. E. Dickinson (1989), How will changes in carbon dioxide and methane modify the mean structure of the mesosphere and thermosphere?, J. Geophys. Res., 16(12), 1441-1444.

Stark, J. P. W., and G. G. Swinerd (2003), Mission analysis, in Spacecraft Systems Engineering, 3rd ed., edited by P. Fortescue et al., pp. 111-167, John Wiley, Chichester, U. K.

United Nations Committee on the Peaceful Uses of Outer Space (UN COPUOS) (2010), Space debris mitigation guidelines of the Committee on the Peaceful Uses of Outer Space, Rep. 09-88517, New York.

H. G. Lewis, R. J. Newland, A. Saunders, and G. Swinerd, Astronautics Research Group, Faculty of Engineering and the Environment, University of Southampton, Southampton SO17 1BJ, UK. (hglewis@soton.ac.uk; r.j. newland@soton.ac.uk; a.saunders@soton.ac.uk; ggs@soton.ac.uk) 